\title{
Nanomechanical sensing of the endothelial cell response to anti-inflammatory action of I-methylnicotinamide chloride
}

This article was published in the following Dove Press journal:

International Journal of Nanomedicine

31 July 2013

Number of times this article has been viewed

\section{AM Kolodziejczyk' \\ GD Brzezinka' \\ K Khurana ${ }^{1,2}$ \\ M Targosz-Korecka' \\ M Szymonski'}

'Research Centre for NanometerScale Science and Advanced Materials, NANOSAM, Faculty of Physics, Astronomy and Applied Computer Science, Jagiellonian University, Krakow, Poland; ${ }^{2}$ Centre for Environmental Risk Assessment and Remediation (CERAR), University of South Australia, Australia
Correspondence: Marek Szymonski Faculty of Physics, Jagiellonian University, Reymonta St No 4, 30-059 Krakow, Poland

Tel +48 I2 6635560

Fax +48 I2 6337086

Email ufszymon@cyf-kr.edu.pl
Background: There is increasing evidence that cell elastic properties should change considerably in response to chemical agents affecting the physiological state of the endothelium. In this work, a novel assay for testing prospective endothelium-targeted agents in vitro is presented.

Materials and methods: The proposed methodology is based on nanoindentation spectroscopy using an atomic force microscope tip, which allows for quantitative evaluation of cell stiffness. As an example, we chose a pyridine derivative, 1-methylnicotinamide chloride (MNA), known to have antithrombotic and anti-inflammatory properties, as reported in recent in vivo experiments.

Results: First, we determined a concentration range of MNA in which physiological parameters of the endothelial cells in vitro are not affected. Then, cell dysfunction was induced by incubation with tumor necrosis factor-alpha (TNF- $\alpha$ ) and the cellular response to MNA treatment after TNF- $\alpha$ incubation was studied. In parallel to the nanoindentation spectroscopy, the endothelium phenotype was characterized using a fluorescence spectroscopy with F-actin labeling, and biochemical methods, such as secretion measurements of both nitric oxide (NO), and prostacyclin $\left(\mathrm{PGI}_{2}\right)$ regulatory agents.

Conclusion: We found that MNA could reverse the dysfunction of the endothelium caused by inflammation, if applied in the proper time and to the concentration scheme established in our investigations. A surprisingly close correlation was found between effective Young's modulus of the cells and actin polymerization/depolymerization processes in the endothelium cortical cytoskeleton, as well as NO and $\mathrm{PGI}_{2}$ levels. These results allow us to construct the physiological model of sequential intracellular pathways activated in the endothelium by MNA.

Keywords: endothelium, cell stiffness, 1-methylnicotinamide chloride, atomic force microscopy, nanomechanical sensing

\section{Introduction}

The structure and functional properties of the endothelium are integral in controlling various physiological processes in the human body, such as homeostasis, inflammation, leukocyte adhesion, or the control of vasomotor tone. ${ }^{1}$ Consequently, dysfunction of the endothelium can lead to many serious diseases that can affect a large number of people. Endothelial cells, similar to muscle or red blood cells, undergo constant squeezing and stretching from the environment due to their function and localization. Therefore, the elasticity of endothelial cells plays a key role in the maintenance of proper blood vessel flow, vascular pressure regulation, and other endogenous functions. ${ }^{1,2}$

In our recent work, an atomic force microscope (AFM) tip sensor was successfully used for characterization of the endothelium inflammatory state by means of 
so-called nanoindentation spectroscopy. ${ }^{2}$ This novel experimental technique allows for quantitative determination of the mechanical properties at the single-cell level..$^{2-4}$ Over the last decade, there have been several studies involving the use of AFM as an imaging tool and/or a mechanical nanosensor for characterization of endothelial cells. ${ }^{5-11}$ Kusche-Vihrog et al reported a significant increase of the human umbilical vein endothelial cell volume and surface area after the application of aldosterone, which was associated with an increased expression of epithelial sodium channel and sodium transport disturbance. ${ }^{5}$ On the other hand, the use of aldosterone antagonists - spironolactone and amiloride - reversed the effect of aldosterone. Oberleithner et al examined the impact of steroid hormones, such as estradiol and aldosterone, on the volume and stiffness of endothelial cells. ${ }^{6}$ Additionally, they found a striking relationship between stiffness and nitric oxide (NO) production by endothelial cells. ${ }^{10,11}$

The results mentioned above encouraged us to establish a working protocol to test the effect of endothelial active pharmaceutics on the mechanical properties at a single-cell level and determine the relationship between the cell elasticity parameter (effective Young's modulus) and the physiological state of the endothelium. In our work, we used a prospective endothelial agent, 1-methylnicotinamide chloride (MNA), which is applied on the endothelial cells under different stages of the inflammatory process caused by tumor necrosis factor-alpha (TNF- $\alpha$ ). MNA is a metabolite of nicotinamide, which is formed in the liver by nicotine N-methyltransferase. It was found by Gebicki et $\mathrm{al}^{12}$ and Wozniacka et $\mathrm{al}^{13}$ that topically applied MNA has a remarkable therapeutic effect on several inflammatory skin diseases, including rosacea, acne vulgaris, skin burns, and wound healing. Later, Chlopicki et $\mathrm{al}^{14}$ demonstrated the anti-inflammatory and antithrombotic potential of MNA in in vivo experiments.

The present work intends to verify whether the mechanical response of human dermal microvascular endothelial cells (HMECs) to incubation with MNA, as measured using nanoindentation spectroscopy with an AFM tip, could be effectively used as a bioindicator of the physiological state of the cells. Therefore, we investigated the extent of the anti-inflammatory effect of MNA on the early stage of inflammation triggered by incubation with TNF- $\alpha$ for 1 hour. Using F-actin staining for optical fluorescence microscopy we could demonstrate that the elasticity changes of the cells are connected to the actin cytoskeleton rearrangement. Additional information on the molecular mechanism of MNA action on endothelium is provided by performing measurements of NO and prostacyclin $\left(\mathrm{PGI}_{2}\right)$ production. The close correlation between the fluorescence microscopy results, the data obtained with the biochemical methods, and the elasticity measurements demonstrate that the effective elasticity modulus could be successfully used for diagnostics of the endothelium phenotype in an in vitro model. The application of the nanoindentation method confirms that MNA can act as a therapeutic agent at a single-cell level.

\section{Materials and methods Cell culture}

HMECs were obtained from the Centers for Disease Control and Prevention (Atlanta, GA, USA). L-glutamine, fetal bovine serum, human epidermal growth factor, and antibiotics used (penicillin, streptomycin, fugison) were purchased from Invitrogen (Life Technologies, Carlsbad, CA, USA). The MCDB medium, Dulbecco's Phosphate-Buffered Saline (DPBS 1X pH 7.4), trypsin (0.25\%), hydrocortisone, and TNF- $\alpha$ were purchased from Sigma-Aldrich (St Louis, MO, USA). MNA in the form of 1-methylnicotinamide chloride was kindly provided by Professor Jerzy Gebicki, Lodz University of Technology (Lodz, Poland). The cell culturing method for HMECs was as previously described. ${ }^{8}$ MCDB medium supplemented with $10 \%$ serum, $1 \%$ L-glutamine, $2 \%$ hydrocortisone, $0.001 \%$ epidermal growth factor, and $0.1 \%$ antibiotics (penicillin, streptomycin, and fungisan) was used. Cells were seeded on the culture flasks and incubated for 3 days at $37^{\circ} \mathrm{C}$, followed by rinsing with DPBS ( $\mathrm{pH} 7.4$ ). Briefly, the cells were trypsinized at $37^{\circ} \mathrm{C}$ to detach the adherent cells from the flask until most of the cells were collected. Next, $2 \mathrm{~mL}$ serum and $5 \mathrm{~mL}$ MCDB medium were added to the flask to neutralize the trypsin. The prepared solution was then transferred to a $15 \mathrm{~mL}$ sterile centrifuge tube for centrifugation at $1200 \mathrm{rpm}$ for 5 minutes and resuspended in fresh growth medium. All of the solvents used were laboratory (suitable for general laboratory applications) and American Chemical Society (high-quality) grade. Each product was used without any additional purification.

\section{Preparation of samples}

Poly-L-lysine glass plates $(1 \mathrm{~cm} \times 1 \mathrm{~cm})$ were rinsed with ethanol and sterilized with ultraviolet light in a laminar chamber for 30 minutes. Then, HMECs from the fourth to seventh passage were seeded on the plates at a concentration of $10^{5}$ cells $/ \mathrm{mL}$ for 48 hours in a $5 \% \mathrm{CO}_{2}$ atmosphere at $37^{\circ} \mathrm{C}$.

The inflammatory effect of TNF- $\alpha$ on HMECs was carefully investigated in our previous work. ${ }^{2}$ The current study took advantage of the previous investigation by exposing the cells to a TNF- $\alpha$ concentration of $10 \mathrm{ng} / \mathrm{mL}$ for additional 
incubation periods $(0.5,2,4$, and 20 hours, while previously the results were published for $1,3,6,12$, and 24 hours) to clearly determine the strong inflammatory phase triggered by TNF- $\alpha$. For each incubation period, a cytokine-unexposed sample was prepared in parallel to the reference. TNF- $\alpha$ was dissolved in fresh medium to a final concentration of $10 \mathrm{ng} /$ $\mathrm{mL}$. The loosely bound cells were removed from the substrate by rinsing with fresh medium heated to $37^{\circ} \mathrm{C}$. In accordance with the previous results, the strongest inflammatory response was obtained following a 1-hour incubation with TNF- $\alpha$; thus, this phase was chosen as an in vitro model of dysfunction by which to study the action of MNA. Prior to the investigation of the MNA anti-inflammatory potential, the effect of MNA alone on healthy cells was characterized to determine the appropriate concentration. Cells were directly exposed to MNA at concentrations of $0.1 \mu \mathrm{M}$ and $10 \mu \mathrm{M}$ for various incubation periods $(1,3,6,12$, or 24 hours). Elasticity measurements revealed that an MNA concentration of $0.1 \mu \mathrm{M}$ was the most suitable (see "Results" section). Motivated by the latter observation, it was found necessary to investigate the anti-inflammatory action of MNA at concentrations of $0.1 \mu \mathrm{M}, 0.2 \mu \mathrm{M}$, and $0.05 \mu \mathrm{M}$.

The cells were exposed to TNF- $\alpha$ for 1 hour and then incubated with MNA for various time periods $(1,3,6,12$, or 24 hours). The cells exposed to TNF- $\alpha$ only were used as a reference for the combined samples (TNF- $\alpha$ and MNA) for the corresponding incubation period. The elasticity, NO production, and fluorescent staining of the actin cytoskeleton were investigated to determine the therapeutic behavior of MNA.

\section{Elasticity measurements}

Nanoindentation spectroscopy using an AFM tip is one of the most versatile techniques for the determination of mechanical properties at the nanometer scale. Elastic properties were characterized with the use of a NanoScope IIIa Multimode ${ }^{\mathrm{TM}}$ SPM (Veeco, Plainview, NY USA) with soft triangular cantilevers (MLCT multilever, spring constant approximately $0.01 \mathrm{~N} / \mathrm{m}$; Veeco). The tip radius and geometry were determined by scanning a TGT1 (NT-MDT, Moscow, Russia) spike grid standard in contact mode and deconvoluted in SPIP software (SPIP 5; Image Metrology A/S, Hørsholm, Denmark). The spring constant of all cantilevers used was calculated from the thermal oscillations of an unloaded lever in air ${ }^{15}$ and analyzed using the NanoScope 6.1 software (Veeco). Prior to measurements, a calibration curve was acquired on a pure glass plate immersed in medium. The samples were placed on a piezo scanner and measured without fixation inside a liquid cell in medium prewarmed to $37^{\circ} \mathrm{C}$. First, the topography of the surface was recorded in AFM contact mode. Then, a $7 \mu \mathrm{m} \times 7 \mu \mathrm{m}$ region (average size) was selected in the central part of the cell, and 144 force-distance curves were collected on the rectangular grid of points. For each sample, at least six different cells were characterized. This study employed the Hertz-Sneddon model ${ }^{16}$ because it offers a reasonable fit to the acquired force-indentation curves. The elasticity parameter $E$ (effective Young's modulus) was calculated for every force-indentation curve by applying the paraboloidal tip model:

$$
F(\delta)=\frac{4}{3} \frac{E}{1-v^{2}} \sqrt{R} \delta^{3 / 2},
$$

where $R$ is the tip radius and the Poisson ratio $(v)$ is assumed to be 0.5 (ie, isotropic elastic properties for small deformations).

The elasticity parameter reflects the cell stiffness, with a lower elasticity parameter indicating a softer cell. For each experimental point (ie, reference cells or cells incubated with investigated agent for a defined incubation period), cumulative histograms were prepared (see Supplementary material). Each histogram is based on all the cells analyzed for a given sample. Force-indentation curve data analysis was as described in our previous work. ${ }^{2}$

The relative change in the elasticity parameter $(\Delta E)$ was calculated for all the experimental points for treated cells (TNF- $\alpha$ or MNA) compared to the control cells for the corresponding incubation period. By fitting the log-normal function, the relative changes in the elasticity parameter were calculated in comparison to the reference cells incubated on the glass for the same time period. In order to compare the data, $\Delta E$ is described by the following equation:

$$
\Delta E=\frac{\bar{E}_{i_{-} \text {agent }}-\bar{E}_{i_{-} \text {control }}}{\bar{E}_{i_{-} \text {control }}},
$$

where $\bar{E}_{i_{-} \text {control }}$ and $\bar{E}_{i_{-} \text {agent }}$ are the median values of the elasticity parameter $E$ for control cells and cells incubated with agents, respectively.

The distribution of the data within each group was tested against log-normal distribution using the KolmogorovSmirnov test at the level $P=0.05$. Additionally, the statistical analysis of the data was performed using analysis of variance to determine differences between means for each group and the control group at the levels $P<0.05$ and $P<0.01$.

\section{Fluorescence microscopy}

Fluorescence microscopic images play a key role in studying the cytoskeletons of cells. They provide us with detailed information about the F-actin and globular G-actin 
(monomeric subunits) content of the microfilaments. The change in the F-actin content results in the modification of the elastic properties, typically followed by the modification of the cell shape. Fluorescence microscopy provides support for observations based on the results of elasticity measurements. To visualize the cytoskeletal reorganization triggered by TNF- $\alpha$ or MNA, F-actin fibers were stained with Alexa Fluor 488 (green). The fluorescent stains (phallotoxine, Alexa Fluor 488 phalloidin) were purchased from Invitrogen (Warsaw, Poland). The poly-L-lysine glass slides were obtained from Thermo Fisher Scientific (Waltham, MA, USA).

Prior to staining, the cells were rinsed with warm phosphate-buffered solution (PBS). The cells were fixed for 10 minutes in $2 \%$ formaldehyde in a PBS solution, rinsed two times with PBS, permeabilized for 4 minutes in $0.1 \%$ Triton X (Sigma-Aldrich) and rinsed twice more with PBS. The cells were then incubated with PBS and $1 \%$ bovine serum albumin (BSA). Next, Alexa Fluor 488 (10 $\mu \mathrm{L} / 500 \mu \mathrm{L}$ PBS) was used to stain the F-actin fibers for 16 minutes at room temperature. Following staining, the excess dye was removed by rinsing two times with PBS.

The stained cell samples were prepared for fluorescence imaging and excited with an Olympus X-Cite Q120 lamp (Olympus Corporation, Tokyo, Japan). They were transmitted by U-MWIB2 (phallotoxine) filters and visualized through an Olympus IX71 with a $20 \times$ objective. The images were recorded and then processed using Olympus CellSense software (version 1.41).

\section{Griess method}

The endothelium actively participates in, and is an important organ affected by, the systemic inflammatory process. Under the influence of an inflammatory agent, endothelial functions reorganize to increase inflammatory mediator secretion and the activity of adhesion molecules. The proinflammatory response of the endothelium is closely associated with changes in the production of vasoprotective mediators, such as NO. NO is an unstable molecule that is quickly metabolized into the more stable compounds nitrite $\left(\mathrm{NO}_{2}^{-}\right)$and nitrate $\left(\mathrm{NO}_{3}^{-}\right)$. The initial concentration of $\mathrm{NO}$ can be indirectly measured by determining the concentrations of nitrite and nitrate. ${ }^{17}$ Briefly, $100 \mu \mathrm{L}$ of solution was obtained from the supernatants of the HMEC culture and incubated with an equal volume of Griess reagent $(0.35 \%$ 4-4-aminophenyl sulfone, $0.1 \%$ N-(1-naphthyl) ethylenediamine dihydrochloride in $1 \mathrm{M}$ hydrochloric acid) at room temperature for 10 minutes. The absorbance at $550 \mathrm{~nm}$ was measured, and the nitrite concentration was calculated from a sodium nitrite standard curve. ${ }^{17}$ The percentage changes in the production of NO following TNF- $\alpha$ exposure with regard to the respective control samples were calculated.

\section{$\mathrm{PGI}_{2}$ production measurements}

The measurement of $\mathrm{PGI}_{2}$ release is a standard method for the evaluation of the physiological state of the endothelium. In vivo, it is typically measured using blood samples through the absorbance measurement of 6-keto-prostaglandin F1 $\alpha$ (PGF1 $\alpha$ ), which is the direct metabolite of $\mathrm{PGI}_{2}$. For the quantitative analysis of 6-keto-PGF1 $\alpha$, we investigated with the enzyme-linked immunosorbent assay method. In the presented work, the $\mathrm{PGI}_{2}$ level provides information about the activated intracellular pathways. The in vitro release of $\mathrm{PGI}_{2}$ was estimated by collecting the supernatants of HMECs cultured in controlled conditions.

Samples incubated with TNF- $\alpha$ only, MNA only, and combination of MNA and TNF- $\alpha$ were investigated. Supernatants were assayed directly using an EIA Kit (Enzo Life Sciences, Warsaw, Poland). The supernatants were then acidified with $0.1 \mathrm{M}$ hydrogen chloride and incubated for 15 minutes at $4^{\circ} \mathrm{C}$. After centrifugation, the liquid phase was applied on a $\mathrm{C}_{18}$ reverse phase extraction column, previously washed with $10 \mathrm{~mL}$ ethanol followed by $10 \mathrm{~mL}$ deionized water. Later, the column was washed sequentially with $10 \mathrm{~mL}$ water, $10 \mathrm{~mL}$ $15 \%$ ethanol, and $10 \mathrm{~mL}$ hexane. Finally, the sample was eluted from the column by the addition of $10 \mathrm{~mL}$ ethyl acetate. Next, the sample was dried in the nitrogen stream and $250 \mu \mathrm{L}$ of assay buffer was added. The sample was then mixed by vortex for 5 minutes at room temperature.

\section{Results and discussion}

\section{Cell elasticity changes indicate the} physiological state of the endothelium

\section{Characterization of the effect of varying MNA} concentrations

Firstly, the relative changes of the elasticity parameter are used for determination of the appropriate range of concentrations at which the effect of MNA alone does not change the endothelial cell functional properties in a noticeable way. The median values of the elasticity parameters determined from their cumulative distributions are presented in Figure 1. Additionally, F-actin staining fluorescence images for various incubation periods with two selected concentrations of MNA are presented in Figure 2.

Endothelial cells incubated with $10 \mu \mathrm{M}$ MNA show a relatively high increase in the elasticity parameter for longer 


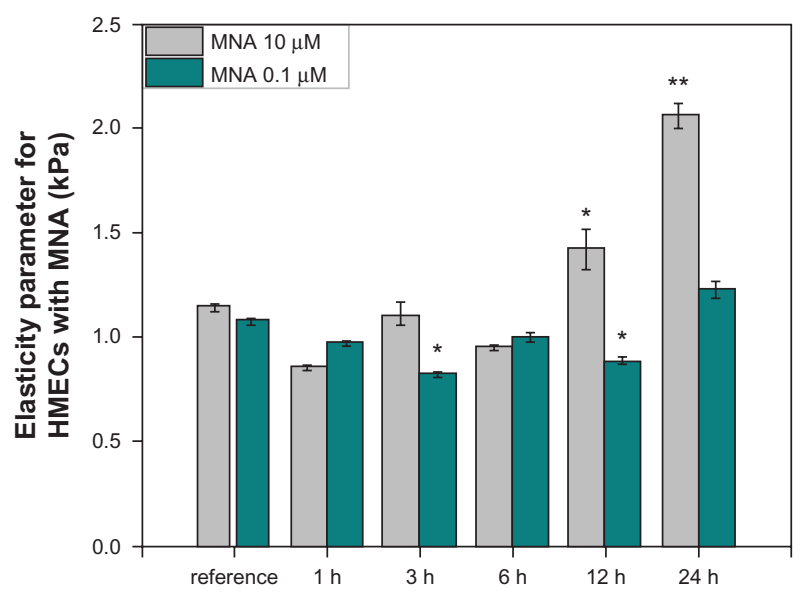

Figure I Comparison of the elasticity parameter for two concentrations of MNA. Notes: An increased elasticity parameter at 12 and 24 hours (h) was observed for $10 \mu \mathrm{M}$ of MNA. The statistically significant differences from the native samples are $* P<0.05$ and $* * p<0.01$.

Abbreviations: HMEC, human dermal microvascular endothelial cell; MNA, I-methylnicotinamide chloride.

periods of incubation (12, 24 hours). These results are in agreement with the optical fluorescence features characteristics for actin cytoskeleton reorganization (Figure 2D and E). Exposing the endothelium to MNA at the higher concentration causes polymerization of the G-actin, and strong, clearly visible, parallel F-actin fibers are present. On the other hand, lowering the concentration of MNA by two orders of magnitude $(0.1 \mu \mathrm{M})$ results in only slight fluctuations of the elasticity parameter, mostly at the level just below the reference. The latter observation is confirmed by a decrease in the F-actin content (Figure 2B and $\mathrm{C}$ ). It is clear, therefore, that the incubation of endothelial cells with $0.1 \mu \mathrm{M}$ of MNA does not induce any visible changes in the phenotype of the cells. On the other hand, the fluorescent staining of the F-actin distribution reveals a minor depolymerization of fibers in comparison to the reference sample. Hence, the $0.1 \mu \mathrm{M}$ concentration of MNA is selected for further investigation of its anti-inflammatory potential. Additionally, further characterization of the dose dependence is performed in the following section on MNA, ie, the concentrations of $0.05 \mu \mathrm{M}$ and $0.2 \mu \mathrm{M}$ are used.

\section{Effect of MNA against inflammation in the endothelium} In our previous work, ${ }^{2}$ the in vitro inflammatory model was established based on TNF- $\alpha$ proinflammatory cytokine. It was found that incubation of endothelial cells with the cytokine results in a two-stage response, as revealed by the changes in cell elasticity (Figure 3A). The strongest inflammatory phase is observed 1 hour after the application of TNF- $\alpha$, and hence it was chosen for the study of the anti-inflammatory potential of MNA. In Figure 3, the relative elasticity parameter changes of the cells sensitized with TNF- $\alpha$ for 1 hour and subsequently incubated for 1,3 , 6,12 , and 24 hours with different concentrations of MNA are presented $(0.05 \mu \mathrm{M}$ [Figure 3B], $0.1 \mu \mathrm{M}$ [Figure 3C], $0.2 \mu \mathrm{M}$ [Figure 3D]). For the lowest concentration of MNA $(0.05 \mu \mathrm{M})$, the inflammatory response of the cells is visible
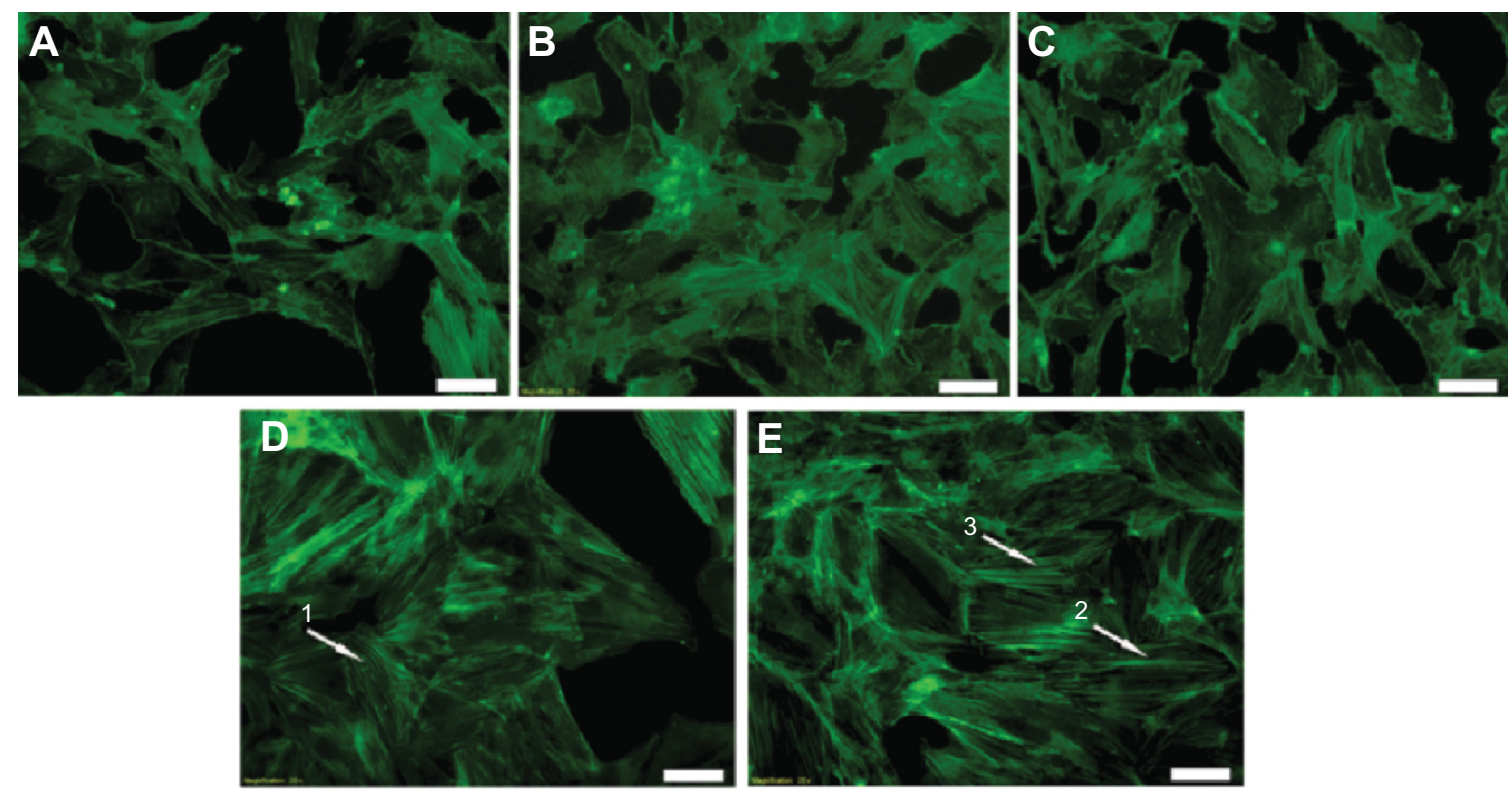

Figure 2 Fluorescent microscopy cell images showing the differences in the thickness and distribution of F-actin fibers in different MNA incubations.

Notes: (A) Unexposed cells; (B) $0.1 \mu$ M (12 hours); (C) $0.1 \mu \mathrm{M}$ (24 hours); (D) $10 \mu \mathrm{M}$ (12 hours); (E) $10 \mu \mathrm{M}$ (24 hours). Arrows indicate the polymerized parallel F-actin fibers. Scale bars: $50 \mu \mathrm{m}$.

Abbreviations: F-actin, filamentous actin; MNA, I-methylnicotinamide chloride. 

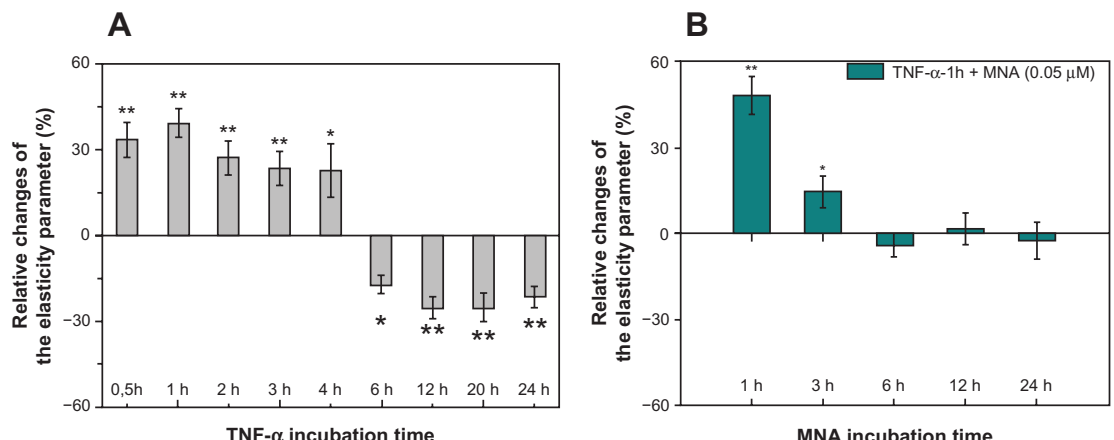

\section{C}
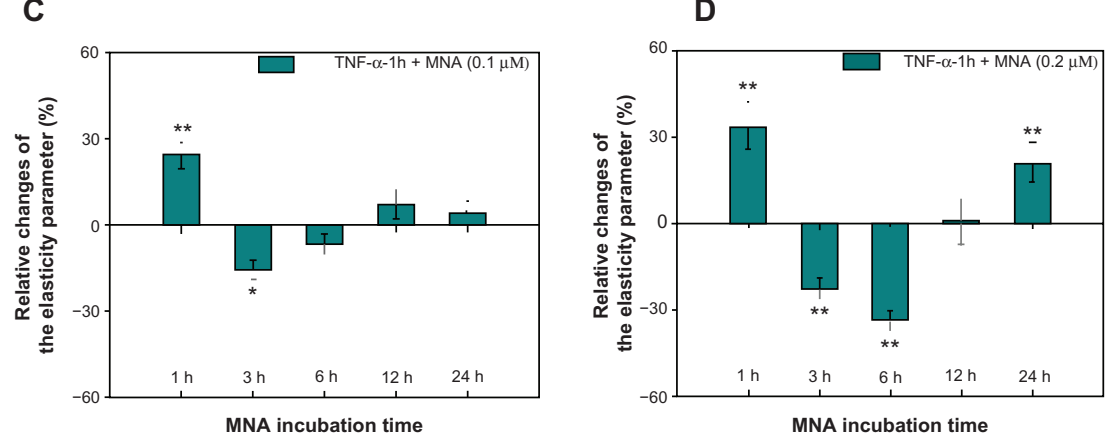

Figure 3 The relative changes of cell elasticity parameter.

Notes: (A) Cells incubated with TNF- $\alpha$ for various periods (gray bars); (B-D) cells incubated with TNF- $\alpha$ (I hour) followed by MNA (I, 3, 6, I2, and 24 hours (h); blue bars) at different MNA concentrations $(0.05 \mu \mathrm{M}, 0.1 \mu \mathrm{M}$, and $0.2 \mu \mathrm{M}$, respectively). The statistically significant differences from the native samples are $* P<0.05$ and $* * P<0.01$. Abbreviations: TNF- $\alpha$, tumor necrosis factor-alpha; MNA, I-methylnicotinamide chloride.

only up to 6 hours. Longer incubation periods reveal the elasticity of cells close to the reference level. Increasing the drug concentration twice $(0.1 \mu \mathrm{M})$ resulted in a drop in elasticity after 1 hour incubation in relation to the cytokine effect, the reversion of TNF- $\alpha$ influence after 3 hours, and
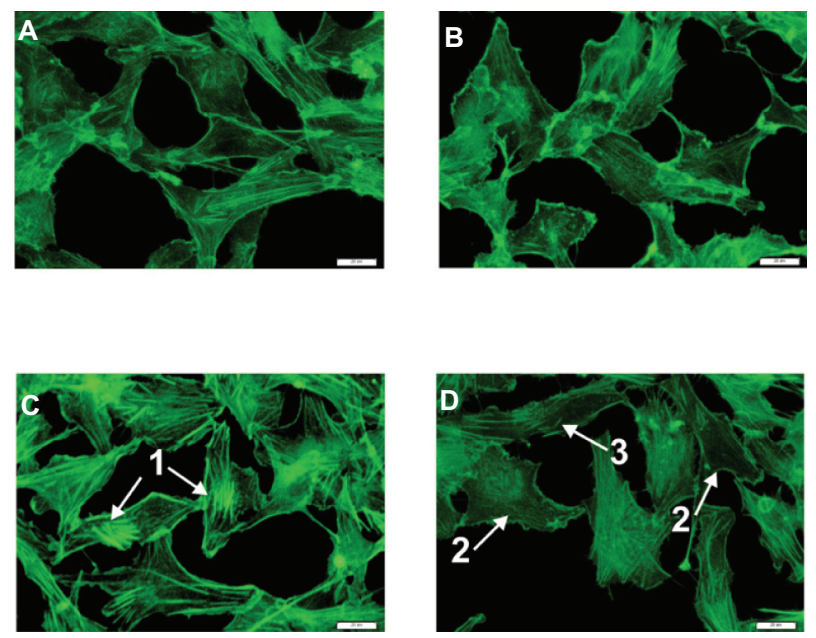

Figure 4 Fluorescent microscopy images.

Notes: (A) Reference; (B) MNA 3 hours; (C) TNF- $\alpha 4$ hours; (D) TNF- $\alpha$ I hour + MNA 3 hours. Arrow I indicates strong fibers, reflecting the polymerization of G-actin; arrow 2 indicates the reference-like cells; and arrow 3 indicates the single F-actin fibers. Scale bars: $20 \mu \mathrm{m}$.

Abbreviations: G-actin, globular actin; TNF- $\alpha$, tumor necrosis factor-alpha; MNA, I-methylnicotinamide chloride; F-actin, filamentous actin. no further changes in relation to the native cells after longer incubation periods. In contrast, the highest concentration of MNA $(0.2 \mu \mathrm{M})$ revealed dramatic changes in the elasticity of the cells. Three and 6 hours after the application of the drug, the elasticity dropped significantly, reverted to the reference level for 12 hours and later increased again for 24 hours. The study has demonstrated the anti-inflammatory response for lower concentrations of the $\operatorname{drug}(0.05 \mu \mathrm{M}, 0.1 \mu \mathrm{M})$. Interestingly, 3 hours of MNA $(0.1 \mu \mathrm{M})$ exposure seems to be crucial, since at this time point, a considerable effect of MNA as an anti-inflammatory agent is acquired.

Based on the observations that the changes in the elasticity parameter are directly connected with the reorganization of the actin cytoskeleton, the fluorescent staining of F-actin fibers was performed. The results presented in Figure 4 depict the F-actin distribution for reference endothelial cells (Figure 4A) and the cells incubated with $0.1 \mu \mathrm{M}$ of MNA for 3 hours (Figure 4B). It can be seen that MNA induces slight depolymerization of the F-actin in comparison to the reference cells. The inflammatory state of the endothelial cells (Figure 4C) is characterized by the presence of short, thick, nonparallel fibers located in the central part of the cell, which is caused by the polymerization of G-actin (Figure 4C). Therefore, it is clear that the action of MNA (Figure 4D) 

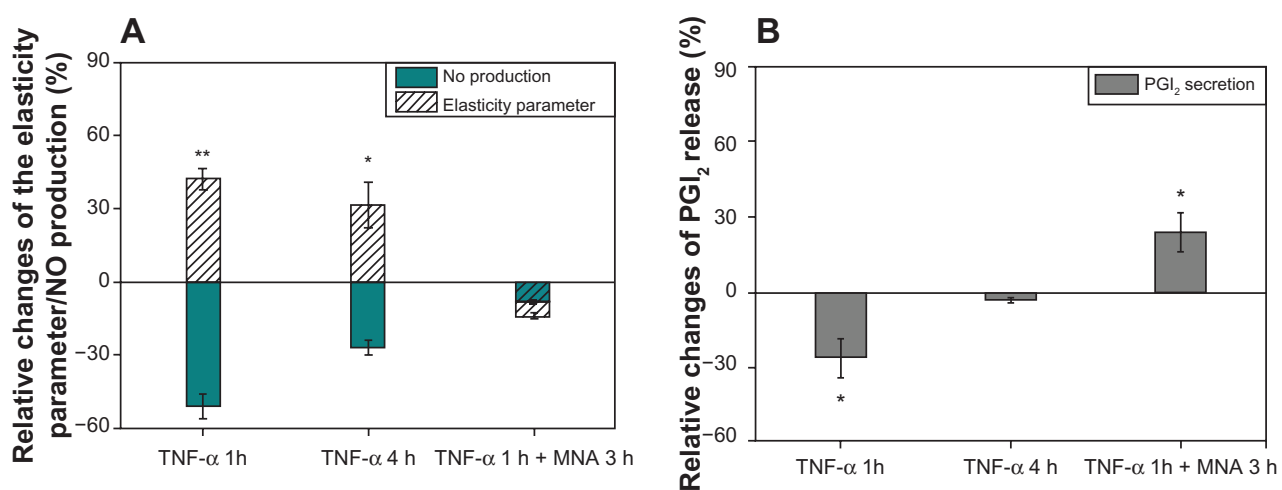

Figure 5 Relative changes of elasticity parameter, $\mathrm{NO}$ production, and $\mathrm{PGI}_{2}$ release.

Notes: (A) Elasticity parameter (white-striped bars)/NO production (blue bars). (B) PGI ${ }_{2}$ release for selected incubation conditions: TNF- $\alpha$ (I hour [h]); TNF- $\alpha$ (4 hours); and TNF- $\alpha$ (I hour) + MNA ( 3 hours). The relative changes of NO production/PGI secretion were calculated compared to the control level (I.83 $\pm 0.4 \mu \mathrm{M}) /(5.97 \pm 0.84 \mathrm{ng} /$ $\mathrm{mL}$ ) (mean $\pm \mathrm{SD}$ ). The statistically significant differences from the native samples are $* P<0.05$ and $* * P<0.01$.

Abbreviations: TNF- $\alpha$, tumor necrosis factor-alpha; MNA, I-methylnicotinamide chloride; NO, nitric oxide; PGI, prostacyclin; SD, standard deviation.

results in the depolymerization of F-actin fibers in relation to the inflamed cells, and only single short fibers are visible. This corresponds with relative changes of the elasticity parameter presented in Figure 3C. On the other hand, an increase in the G-actin content contributes to $\mathrm{NO}$ production in two ways. Firstly, the elevated level of G-actin increases the availability of binding sites for endothelial NO synthase (eNOS). Secondly, it stimulates the transport of L-arginine, which is the main substrate for NO synthesis. ${ }^{18,19}$

\section{Correlations between elasticity changes and biochemical parameters in response to MNA}

As stated in the previous sections, the response of endothelial cells to MNA anti-inflammatory action leads to the attenuation of the elasticity parameter changes with respect to untreated endothelium. To further understand this effect, the elasticity measurements are supplemented with $\mathrm{NO}$ and $\mathrm{PGI}_{2}$ release measurement. In Figure 5, the results obtained for the inflammatory states triggered by 1 and 4 hours of incubation

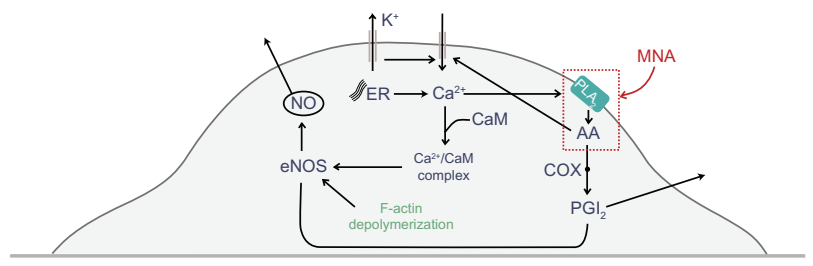

Figure 6 The cycle of processes involved in the intracellular flow of $\mathrm{Ca}^{2+}, \mathrm{NO}$, and $\mathrm{PGI}_{2}$.

Notes: The red rectangle indicates the components that might be directly influenced by MNA.

Abbreviations: AA, arachidonic acid; CaM, calmodulin; COX, cyclooxygenase; eNOS, endothelial nitric oxide (NO) synthase; ER, endoplasmic reticulum; F-actin, filamentous actin; MNA, I-methylnicotinamide chloride; $\mathrm{PGI}_{2}$, prostacyclin; PLA, phospholipase A2. with TNF- $\alpha$ are compared with the cell response following a 3-hour MNA incubation of cells previously exposed to the cytokine for 1 hour, ie, for the same total incubation time. TNF- $\alpha$ applications for 1 and 4 hours result in a distinctive increase of the elasticity parameter, while incubation with MNA leads to a reduction of the elasticity parameter back to a value close to the reference level (Figure 5A). Furthermore, the inflammation caused by incubation with TNF- $\alpha$ leads to a significant reduction in NO production, which again approaches the reference level following the application of MNA for 3 hours (Figure 5A).

Furthermore, it can be seen in Figure 5B that TNF- $\alpha$ greatly reduces the secretion of $\mathrm{PGI}_{2}$ for 1 hour incubation time, whereas for 4 hours of incubation, a rather slight reduction of the secretion is observed. For cells incubated with TNF- $\alpha$ for 1 hour and then MNA for 3 hours, the PGI $_{2}$ level is much higher than for the reference cells. These results confirm that the intracellular pathways induced by MNA are also connected to $\mathrm{PGI}_{2}$ and $\mathrm{NO}$ production by the cells.

\section{Intracellular pathways activated by MNA}

Our investigations demonstrate that MNA induces changes in the concentration of $\mathrm{NO}$ and $\mathrm{PGI}_{2}$. The results are consistent with in vivo investigations by Chlopicki et al, ${ }^{14}$ who suggested that MNA elevates the concentration of arachidonic acid (AA), very likely through the activation of phospholipase $A_{2}$, an enzyme located in the cellular membrane that releases AA from the membrane phospholipids. Based on these observations, we considered possible molecular pathways involved in the MNA action on endothelial cells, schematically shown in Figure 6. It is plausible that the increased level of AA initiates two intrinsic pathways leading to the stimulation of $\mathrm{NO}$ production, which is the marker of anti-inflammatory response. The first pathway is 
connected with the metabolism of AA through the cyclooxygenase to $\mathrm{PGI}_{2}$. This pathway, leading to an increase in the $\mathrm{PGI}_{2}$ level, is consistent with the results presented in Figure 5B. The $\mathrm{PGI}_{2}$ directly activates eNOS, which synthesizes $\mathrm{NO} .^{20}$ In the second pathway, AA directly opens calcium-permeable channels, resulting in an increase in $\mathrm{Ca}^{2+}$ levels. ${ }^{21}$ Calcium ions may create a complex with calmodulin, which binds to eNOS, inducing the NO production. ${ }^{22}$ Additionally, F-actin depolymerization gives rise to the number of eNOS binding sites, which is constituted by the enhanced eNOS activity and elevates NO release. ${ }^{18}$ The application of MNA to inflamed endothelial cells, which present decreased levels of NO, activates the proposed pathways leading to the elevation in NO production up to the level of native cells, as shown in Figure 5A.

\section{Conclusion}

In this work, we demonstrated that cell elasticity could serve as a very sensitive bioindicator of the endothelium physiological state. Since quantitative determination of the cell Young's modulus has become possible by means of nanoindentation spectroscopy using an AFM tip, the elasticity measurement could offer an alternative method for cell diagnostics and testing the cellular activity of various pharmacological agents. This conclusion has been verified by the case study performed with MNA, known to have anti-inflammatory properties in in vivo experiments. The study shows the dose-dependent MNA influence on the elasticity of the cells, confirmed by the F-actin labeling and fluorescence microscopy, as well as by analyzing changes in $\mathrm{NO}$ production and $\mathrm{PGI}_{2}$ release. Furthermore, we propose the physiological mechanism for this MNA activity, based on the biochemical cycle that involves $\mathrm{NO}$ and $\mathrm{PGI}_{2}$ regulatory agents. This mechanism may explain the therapeutic effects of MNA against inflammation of the endothelium in both in vitro and in vivo environments.

\section{Acknowledgments}

MNA was kindly provided by Professor Jerzy Gebicki, Lodz University of Technology. Numerous discussions with Professor Stefan Chlopicki are gratefully acknowledged. Nitrite determination using Griess reagents was performed at the Department of Immunology, Jagiellonian University College of Medicine, Krakow, Poland, courtesy of Dr Rafal Biedron. $\mathrm{PGI}_{2}$ release was measured at the Jagiellonian Center for Experimental Pharmaceutics, Krakow, Poland courtesy of Dr Tomasz Wojcik and Barbara Sitek. This work was supported by the European Union from the resources of the European Regional Development Fund under the Innovative Economy Program (grant coordinated by JCET-UJ, No POIG.01.01.02-00-069/09).

\section{Disclosure}

The authors report no conflicts of interest in this work.

\section{References}

1. Aird WC. Spatial and temporal dynamics of the endothelium. JThromb Haemost. 2005;3(7):1392-1406.

2. Szczygiel AM, Brzezinka G, Targosz-Korecka M, Chlopicki S, Szymonski M. Elasticity changes anti-correlate with NO production for human endothelial cells stimulated with TNF- $\alpha$. Pflugers Arch. 2011;463(3):487-496.

3. Cappella B, Dietler G. Force-distance curves by atomic force microscopy. Surf Sci Rep. 1999;34:1-104.

4. Zuk A, Targosz-Korecka M, Szymonski M. Effect of selected drugs used in asthma treatment on morphology and elastic properties of red blood cells. Int J Nanomedicine. 2011;6:249-257.

5. Kusche-Vihrog K, Sobczak K, Bangel N, et al. Aldosterone and amiloride alter $\mathrm{ENaC}$ abundance in vascular endothelium. Pflugers Arch. 2008;455(5):849-857.

6. Oberleithner H, Riethmüller C, Ludwig T, Hausberg M, Schillers H. Aldosterone remodels human endothelium. Acta Physiol (Oxf). 2006;187(1-2):305-312.

7. Callies C, Fels J, Liashkovich I, et al. Membrane potential depolarization decreases the stiffness of vascular endothelial cells. J Cell Sci. 2011;124(Pt 11):1936-1942.

8. Oberleithner H, Peters W, Kusche-Vihrog K, et al. Salt overload damages the glycocalyx sodium barrier of vascular endothelium. Pflugers Arch. 2011;462(4):519-528.

9. Oberleithner H. Two barriers for sodium in vascular endothelium? Ann Med. 2012;44 Suppl 1:S143-S148.

10. Oberleithner H, Callies C, Kusche-Vihrog K, et al. Potassium softens vascular endothelium and increases nitric oxide release. Proc Natl Acad Sci U S A. 2009;106(8):2829-2834.

11. Oberleithner H, Riethmüller C, Schillers H, MacGregor GA, de Wardener HE, Hausberg M. Plasma sodium stiffens vascular endothelium and reduces nitric oxide release. Proc Natl Acad Sci U SA. 2007;104(41):16281-16286.

12. Gebicki J, Sysa-Jedrzejowska A, Adamus J, Woźniacka A, Rybak M, Zielonka J. 1-Methylnicotinamide: a potent anti-inflammatory agent of vitamin origin. Pol J Pharmacol. 2003;55(1):109-112.

13. Wozniacka A, Wieczorkowska M, Gebicki J, Sysa-Jedrzejowska A. Topical application of 1-methylnicotinamide in the treatment of rosacea: a pilot study. Clin Exp Dermatol. 2005;30(6):632-635.

14. Chlopicki S, Swies J, Mogielnicki A, et al. 1-Methylnicotinamide (MNA), a primary metabolite of nicotinamide, exerts anti-thrombotic activity mediated by a cyclooxygenase-2/prostacyclin pathway. $\mathrm{Br} J$ Pharmacol. 2007;152(2):230-239.

15. Schäffer TE. Calculation of thermal noise in an atomic force microscope with a finite optical spot size. Nanotechnology. 2005;16(6):664-670.

16. Sneddon IN. The relation between load and penetration in the axisymmetric boussinesq problem for a punch of arbitrary profile. Int J Eng Sci. 1965;3(1):47-57.

17. Ding AH, Nathan CF, Stuehr DJ. Release of reactive nitrogen intermediates and reactive oxygen intermediates from mouse peritoneal macrophages. J Immunol. 1988;141(7):2407-2412.

18. Su Y, Edwards-Bennett S, Bubb MR, Block ER. Regulation of endothelial nitric oxide synthase by the actin cytoskeleton. Am J Physiol Cell Physiol. 2003;284(6):C1542-C1549.

19. Ścibior D, Czeczot H. Arginine - metabolism and functions in cardiovascular system. Adv Clin Exp Med. 2005;14(5):1041-1050.

20. Bolego C, Buccellati C, Radaelli T, et al. eNOS, COX-2, and prostacyclin production are impaired in endothelial cells from diabetics. Biochem Biophys Res Commun. 2006;339(1):188-190.

21. Munaron L. Intracellular calcium, endothelial cells and angiogenesis. Recent Pat Anticancer Drug Discov. 2006;1(1):105-119.

22. Fleming I, Busse R. Signal transduction of eNOS activation. Cardiovasc Res. 1999;43(3):532-541. 


\section{Supplementary materials}

A

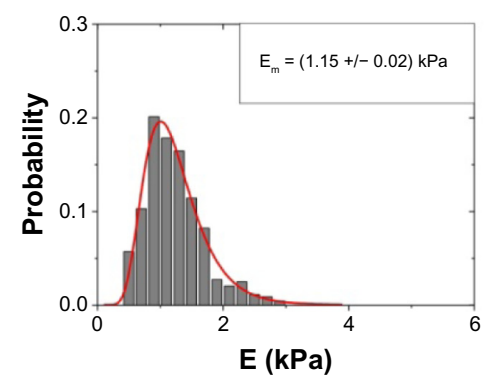

D

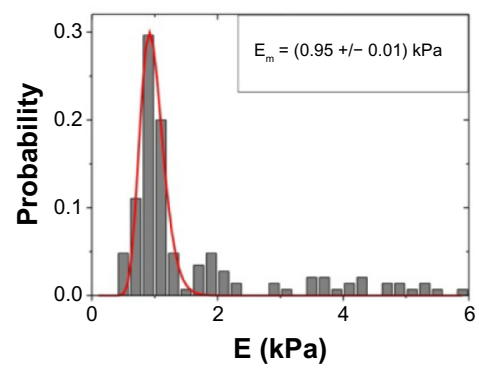

B

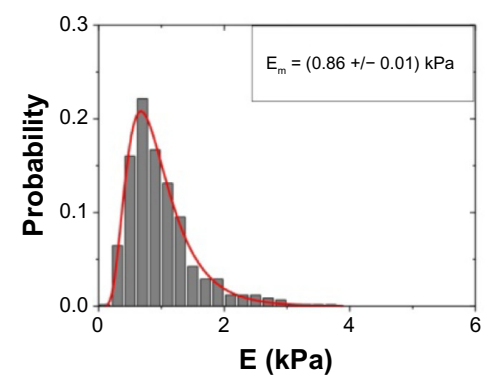

E

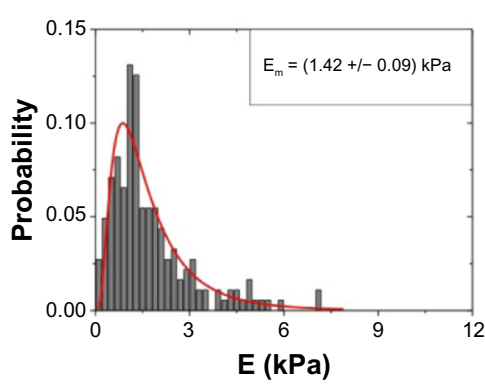

C
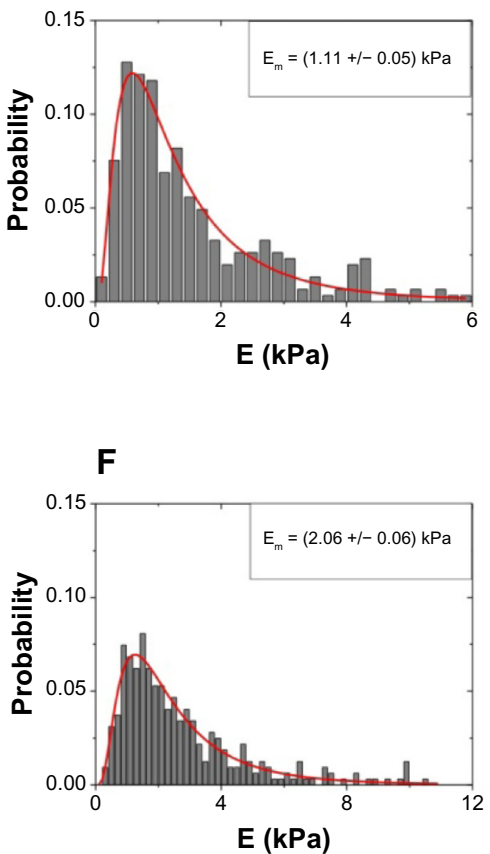

Figure SI Elasticity parameter (E) distributions for reference cells (A) and cells incubated with $10 \mu$ M of MNA for (B) I hour, (C) 3 hours, (D) 6 hours, (E) I2 hours and (F) 24 hours. Log-normal distribution (solid curves), resultant median values and its inaccuracies are presented.

Abbreviation: MNA, I-methylnicotinamide chloride.

A

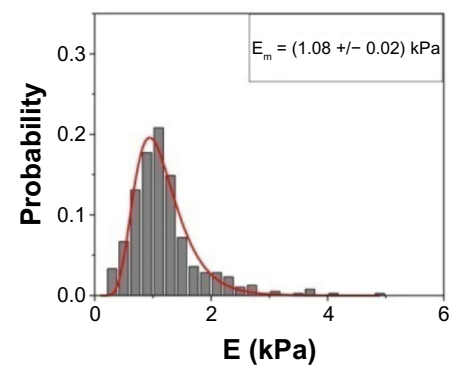

D

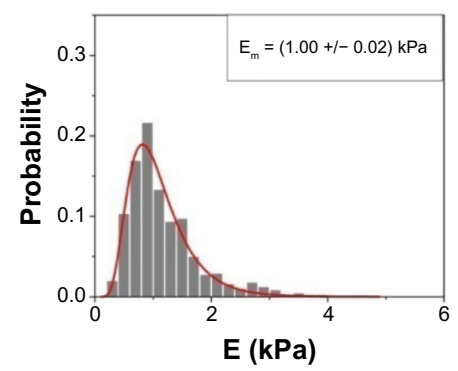

B

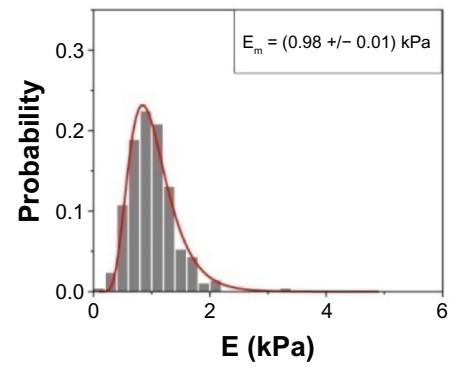

E

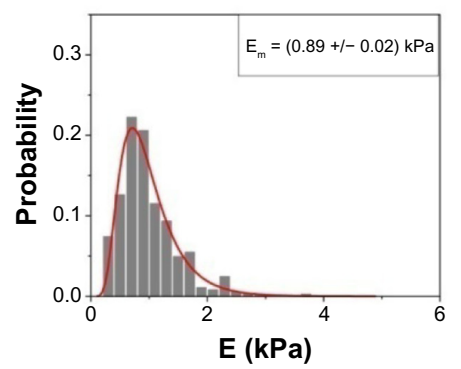

C
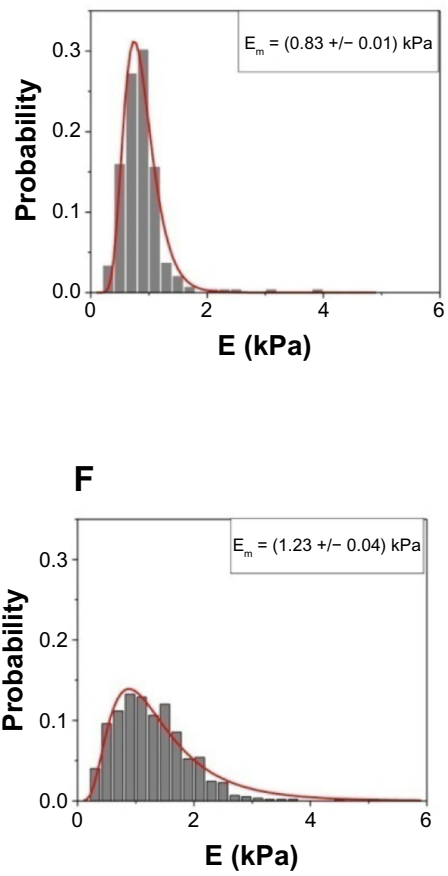

Figure S2 Elasticity parameter (E) distributions for reference cells (A) and cells incubated with $0.1 \mu$ M of MNA for (B) I hour, (C) 3 hours, (D) 6 hours, (E) I 2 hours and (F) 24 hours. Log-normal distribution (solid curves), resultant median values and its inaccuracies are presented.

Abbreviation: MNA, I-methylnicotinamide chloride. 
A

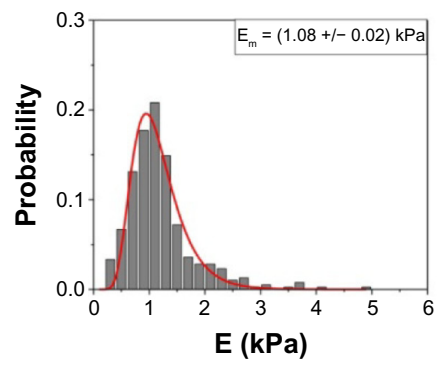

D

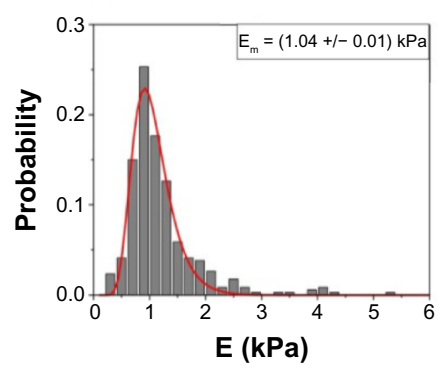

B

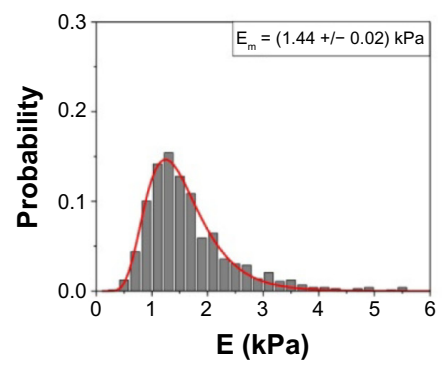

E

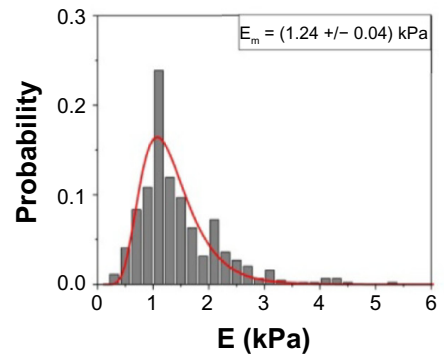

C

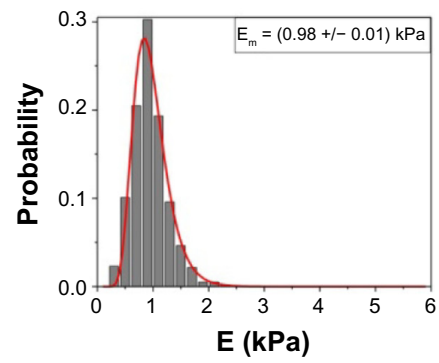

$\mathbf{F}$

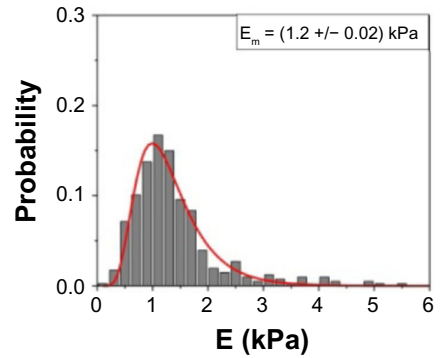

Figure S3 Elasticity parameter (E) distributions for reference cells (A) and cells incubated with TNF- $\alpha$ (I hour) plus $0.1 \mu$ M of MNA for (B) I hour, (C) 3 hours, (D) 6 hours, (E) 12 hours and (F) 24 hours. Log-normal distribution (solid curves), resultant median values and its inaccuracies are presented.

Abbreviations: MNA, I-methylnicotinamide chloride; TNF- $\alpha$, tumor necrosis factor-alpha.

A

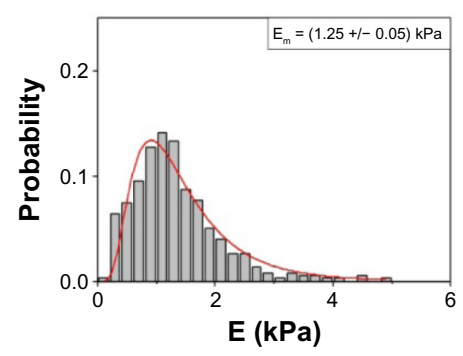

D

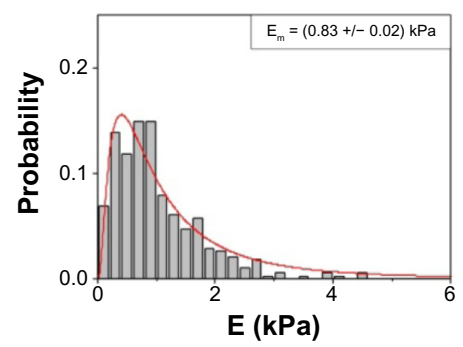

B

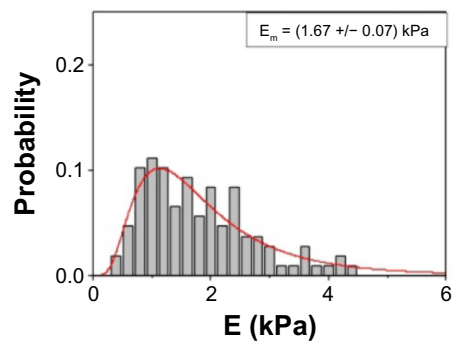

E

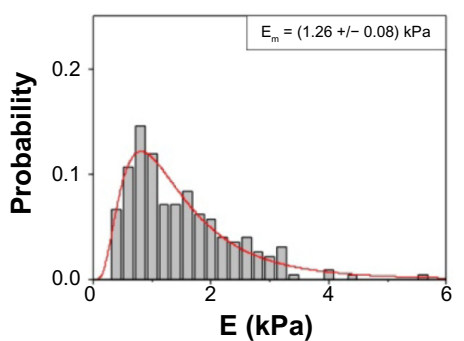

C

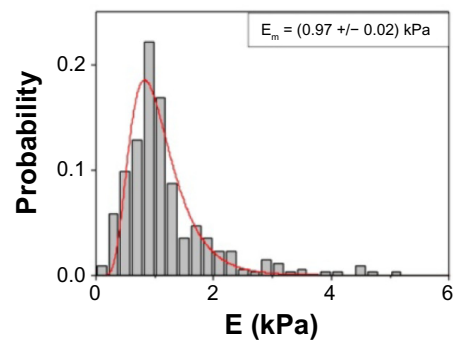

F

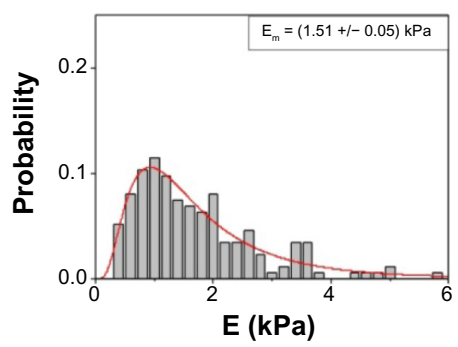

Figure S4 Elasticity parameter (E) distributions for reference cells (A) and cells incubated with TNF- $\alpha$ (I hour) plus $0.2 \mu$ M of MNA for (B) I hour, (C) 3 hours, (D) 6 hours, (E) 12 hours and (F) 24 hours. Log-normal distribution (solid curves), resultant median values and its inaccuracies are presented. Abbreviations: MNA, I-methylnicotinamide chloride; TNF- $\alpha$, tumor necrosis factor-alpha. 
A

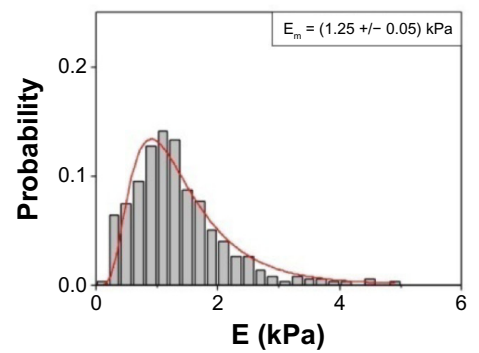

D

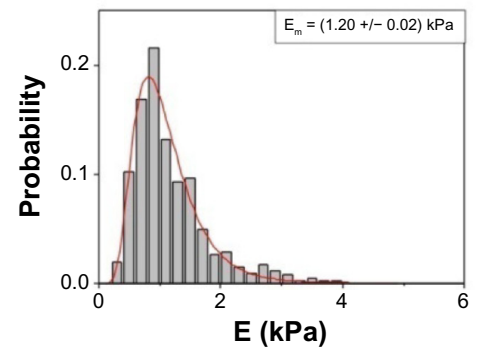

B

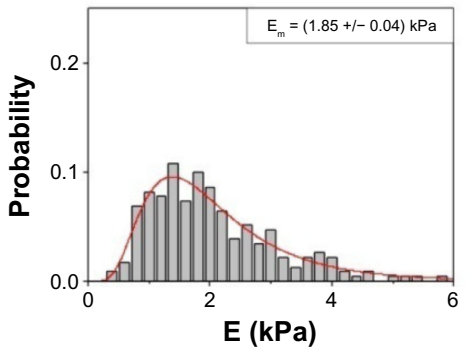

E

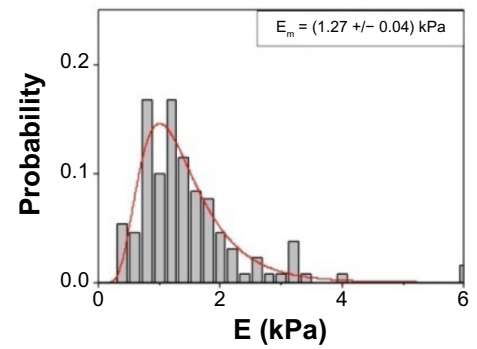

C

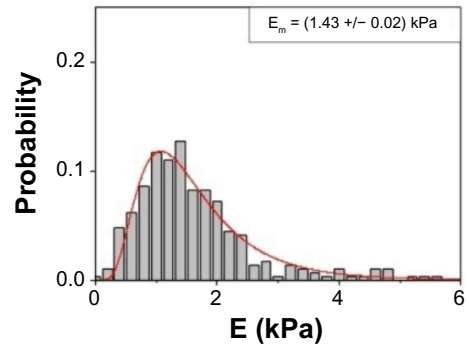

$\mathbf{F}$

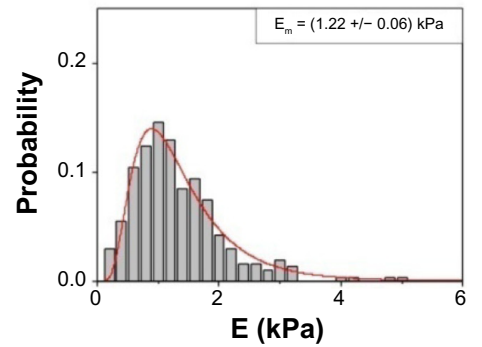

Figure S5 Elasticity parameter (E) distributions for reference cells (A) and cells incubated with TNF- $\alpha$ (I hour) plus $0.05 \mu$ M of MNA for (B) I hour, (C) 3 hours, (D) 6 hours, (E) 12 hours and (F) 24 hours. Log-normal distribution (solid curves), resultant median values and its inaccuracies are presented.

Abbreviations: MNA, I-methylnicotinamide chloride; TNF- $\alpha$, tumor necrosis factor-alpha.

International Journal of Nanomedicine

\section{Publish your work in this journal}

The International Journal of Nanomedicine is an international, peerreviewed journal focusing on the application of nanotechnology in diagnostics, therapeutics, and drug delivery systems throughout the biomedical field. This journal is indexed on PubMed Central,

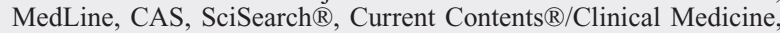

\section{Dovepress}

Journal Citation Reports/Science Edition, EMBase, Scopus and the Elsevier Bibliographic databases. The manuscript management system is completely online and includes a very quick and fair peer-review system, which is all easy to use. Visit http://www.dovepress.com/ testimonials.php to read real quotes from published authors. 